\title{
Inevitable chemical effect of balance gas in low temperature plasma assisted combustion
}

\author{
Ramses Snoeckx* and Min Suk Cha
}

King Abdullah University of Science and Technology (KAUST), Clean Combustion Research Center (CCRC), Physical Science and Engineering Division (PSE), Thuwal 23955, Saudi Arabia.

*corresponding author: ramses.snoeckx@kaust.edu.sa

\begin{abstract}
Electrical discharges (or plasmas) have attracted researchers' attention to improve combustion characteristics. One of its key effects, which is not fully understood yet, is the in-situ production of chemically reactive species. Since most related low temperature kinetic studies to-date have been performed under highly diluted conditions (>99\%), here we present the inevitable and undesirable chemical effect of a balance gas $\left(\mathrm{Ar}, \mathrm{He}, \mathrm{N}_{2}\right)$ on the plasma-chemical kinetics. We employ a zero dimensional plasma-chemical kinetics model in combination with a (detailed and reduced) $\mathrm{H}_{2} / \mathrm{O}_{2} / \mathrm{Ar}$ reaction mechanism. The presented results indicate that (dissociative) quenching of excited (metastable) states dominates the $\mathrm{H}_{2}$ and $\mathrm{O}_{2}$ dissociation processes under highly diluted conditions. Additionally, in the reduced field intensity $(E / N)$ domain, the type and amount of the balance gas significantly alters the fraction of electron energy transferred to the other species in the mixture. Therefore, we propose essential steps for the design of future kinetic studies for plasma assisted combustion.
\end{abstract}

Keywords: plasma chemistry; electrical discharges; excited metastable states; hydrogen 
Non-equilibrium plasma discharges are increasingly studied to enhance combustion processes and reform fuels [1-3]. Electrical discharges can positively affect combustion phenomena, through electron impact reactions leading to the in-situ production of chemically reactive species, such as electrons, radicals, excited species, and ions. Plasma-chemical kinetic models, validated by experiments, are essential to develop a predictive tool for plasma assisted combustion. Following the communities best practices, several low temperature studies with plasma flow reactors have been performed under highly diluted conditions (> $99 \%$ ) with either Argon [4] or Helium [5]. Such highly diluted mixtures limit the temperature increase from the chemical reactions and thus allows an isothermal approximation. The key hypothesis of this approach is the balance gas being chemically inert and not affecting the system, which is reasonably valid for combustion processes. Here we show, however, why there is no chemically inert balance gas in plasma assisted combustion systems, and what the implications of the balance gas' chemical effect are on the development of plasma-chemical kinetic models.

To clarify the effect of the balance gas on the plasma chemistry, we present simulations using our in-house zero-dimensional (0D) plasma-chemical kinetics model (which incorporates the temperature increases due to chemical reactions) and $\mathrm{H}_{2} / \mathrm{O}_{2} / \mathrm{Ar}$ reaction mechanisms. The conditions in our simulations are based on a previous study [4], which investigated the plasmachemical kinetics of $\mathrm{H}_{2} / \mathrm{O}_{2}$ diluted with $\mathrm{Ar}$ (initial species mole percent: $\chi\left(\mathrm{H}_{2}\right)=0.2 \%, \chi\left(\mathrm{O}_{2}\right)=0.3$ $\%$, and $\chi(\mathrm{Ar})=99.5 \%)$ using nano-second discharges. Figure 1 compares our simulations with these previous experimental and modeling results [4].

Our simulations can reasonably well predict the experimental result reported by Tsolas et al. [4]. The difference at the lowest temperatures most probably originates from an overestimation of the $\mathrm{H}_{2}$ conversion in the simulations due to the uncertainties on the thermal mechanism at these temperatures and/or an underestimation in the experiments due to the measurement uncertainties. The improved prediction of the present modeling results most probably stems from the used plasma-chemical kinetic mechanism for Ar. Figure 1 shows the outlet mole fractions of $\mathrm{H}_{2}$ and $\mathrm{O}_{2}$ for various initial temperatures. Full oxidation of $\mathrm{H}_{2}$ occurs above $850 \mathrm{~K}$, while the observed partial conversion at lower temperatures is a result of plasma-chemical reactions. The corresponding reduced field intensity $(E / N)$, defined by the electric field intensity $(E)$ divided by 
the number density of the gas $(N)$, is also depicted in the upper $x$-axis for comparison; showing increased $E / N$ with increased temperature for a fixed $E$.

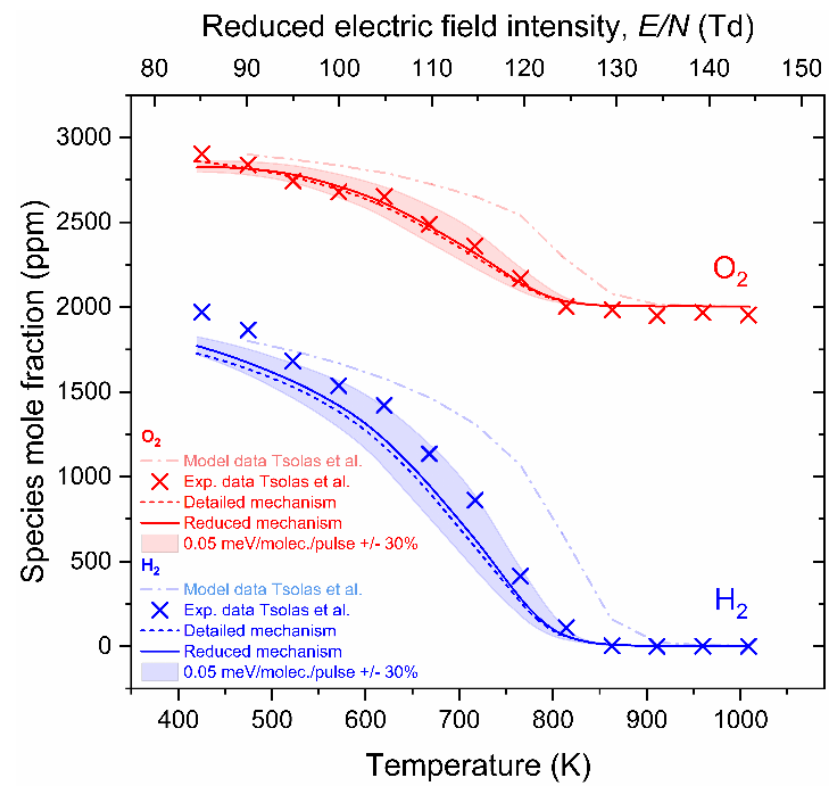

Fig. 1. $\mathrm{H}_{2}$ (blue) and $\mathrm{O}_{2}$ (red) mole fractions as a function of the initial temperature for a mixture of $\chi(\mathrm{Ar})=$ $99.5 \%, \chi\left(\mathrm{H}_{2}\right)=0.2 \%$, and $\chi\left(\mathrm{O}_{2}\right)=0.3 \%, 1 \mathrm{kHz}$ nano-second pulse discharges with $0.05 \mathrm{meV}$ (molecule) $)^{-}$ ${ }^{1}$ (pulse $)^{-1}$ ( $\pm 30 \%$ represented by the shaded areas) as estimated energy deposition [4].

The detailed reaction mechanism (dashed lines) can be drastically reduced (solid lines), while maintaining similar predictions (Fig. 1). Because most of the electron energy is transferred to Ar (Fig. 2a), we intentionally remove all the direct electron impact reactions with $\mathrm{H}$ and $\mathrm{O}$ containing species (e.g., electron impact excitation/dissociation/ionization/attachment), as well as their respective ions and corresponding ion reactions (e.g., electron-ion recombination, ion-ion, and ionneutral reactions). This yields the reduced mechanism with 16 species involved in 75 reactions (see Supplementary material, Table $\mathrm{S} 1$ and S2), consisting of a pure thermal $\mathrm{H}_{2} / \mathrm{O}_{2}$ mechanism (USC mech2.0, 28 reactions) [6] extended with two $\mathrm{O}_{3}$-reactions, a simplified Ar mechanism (30 reactions), and 15 dissociative quenching (or Penning dissociation) reactions of Ar metastable states with $\mathrm{H}_{2}, \mathrm{O}_{2}, \mathrm{H}_{2} \mathrm{O}$ and $\mathrm{O}_{3}$. Interestingly, there is almost no difference between the detailed 
(33 species, 221 reactions) and reduced reaction mechanism, for this highly diluted case, due to the dominant effect of reactive excited metastable species on the chemistry.

The reactivity of excited metastable species is definitely not a new concept. However, to-date the main focus has been on how excited states, such as $\mathrm{O}_{2}\left(\mathrm{a}^{1} \Delta_{\mathrm{g}}\right)$ and $\mathrm{O}\left({ }^{1} \mathrm{D}\right)$, can enhance the combustion process directly [1,2,7,8], as well as on how Ar can enhance it indirectly [1,2,4,7]. Or on how the presence of $\mathrm{N}_{2}$ and its excited states influence the energy distribution, excitation processes, and contributes to gas heating in plasma assisted combustion studies [1,2,8-10]. Here, however, we focus our attention on the flip side of the coin.

These similar predictions between both mechanisms indicate the undesired — but inevitableeffect of the balance gas in plasma assisted combustion, especially with respect to the development of plasma-chemical kinetic models. The excited metastable states of common balance gases (Ar [11], He [12], and $\mathrm{N}_{2}$ [13]) play a crucial role. For the conditions under study with Ar, most of the energetic electrons' energy is transferred to electronically excite Ar, forming highly energetic metastable states, Ar* (Eq. 1). The collisional quenching rates of Ar* with $\mathrm{H}_{2}$ and $\mathrm{O}_{2}$ are four orders of magnitude faster than that with ground state Ar [14,15]. More importantly, collisional quenching of Ar* leads to the dissociation of $\mathrm{H}_{2}$ (Eq. 2) and $\mathrm{O}_{2}$ (Eq. 3).

$$
\begin{aligned}
& \mathrm{e}^{-}+\mathrm{Ar} \rightarrow \mathrm{e}^{-}+\mathrm{Ar}^{*}, \quad \mathrm{Ar}^{*}=\mathrm{Ar}(4 \mathrm{~s}), \operatorname{Ar}(4 \mathrm{p}) \\
& \mathrm{Ar}^{*}+\mathrm{H}_{2} \rightarrow \mathrm{Ar}+\mathrm{H}+\mathrm{H} \\
& \mathrm{Ar}^{*}+\mathrm{O}_{2} \rightarrow \mathrm{Ar}+\mathrm{O}+\mathrm{O}
\end{aligned}
$$

Hence, based on our results, we can conclude that Penning dissociation (Eqs. 2 and 3) prevails over all other direct electron impact and ion reactions with $\mathrm{H}_{2}$ and $\mathrm{O}_{2}$ in a highly diluted mixture. Therefore, this implies that (i) it is impossible to validate whether a reaction mechanism is actually accurate for such a highly diluted case, and (ii) it is impossible to extend a mechanism to other balance gases, due to the difference in reactivity of their excited (metastable) states.

To detail the effect of the balance gas on the plasma physics, we discuss the fraction of the electron energy transferred to each species as a function of $E / N$ (Fig. 2), which follows from the 
electron energy distribution function (EEDF). For more information regarding the importance of both concepts in plasma assisted combustion, we refer to [2,7]. Note that the transferred energy to each species is a summation of all direct electron impact reactions. We use $\mathrm{Ar}, \mathrm{He}$, and $\mathrm{N}_{2}$, with various dilution rates $\left(99.5,90\right.$, and $10 \%$ ), for a fixed $2 / 3$ molar ratio of $\mathrm{H}_{2} / \mathrm{O}_{2}$. The threshold energies (e.g. ionization energy) and cross sections of the electron impact reactions are very different for each (balance) gas. As a result, the respective energy transfer to the balance gas, $\mathrm{H}_{2}$, and $\mathrm{O}_{2}$ varies drastically with a change in (or amount of) the balance gas. For example, at a $90 \%$ dilution rate (Fig. 2b, e, h), the portion of electron energy transferred to $\mathrm{H}_{2}$ and $\mathrm{O}_{2}$ is highest with He, and lowest with $\mathrm{N}_{2}$.

$99.5 \%$

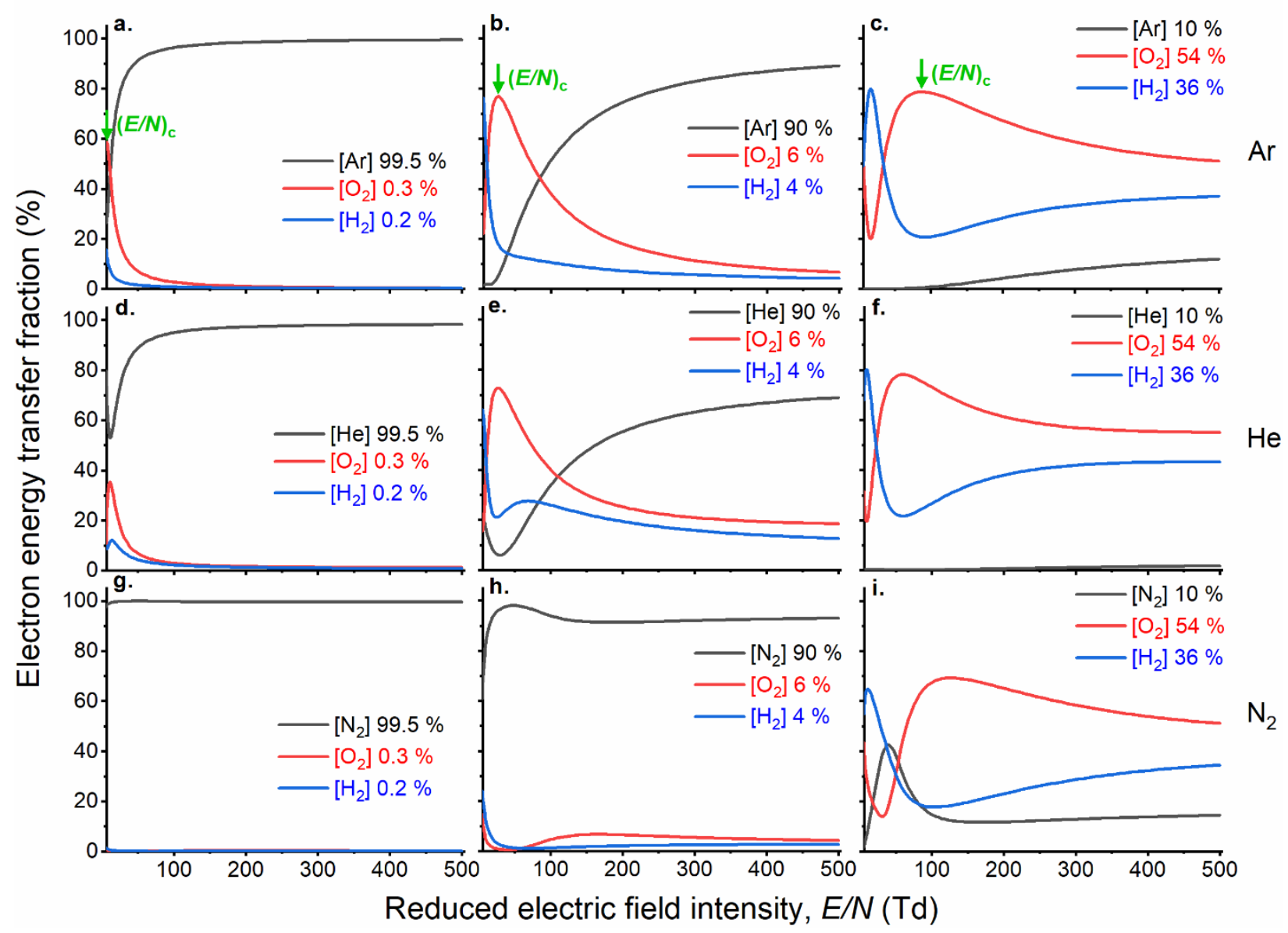

Fig. 2. Fraction of the electron energy transferred to the balance gas (black), $\mathrm{O}_{2}$ (red), and $\mathrm{H}_{2}$ (blue) as a function of $E / N$ for various dilutions. $(E / M)_{c}$ indicates where the energy transfer to $\mathrm{O}_{2}$ is at its maximum. Data is obtained with bolsig + [16] using the Phelps database [17]. 
At the same time, the amount of balance gas also significantly affects the fraction of electron energy transferred to $\mathrm{H}_{2}$ and $\mathrm{O}_{2}$; the fraction increases with decreasing dilution rate (Fig. $2 \mathrm{a} \rightarrow \mathrm{b} \rightarrow \mathrm{c}$ ). Therefore, with decreasing dilution rate we can expect a higher contribution of the direct electron impact reactions with $\mathrm{H}_{2}$ and $\mathrm{O}_{2}$ to the overall plasma assisted oxidation, and vice versa. In addition, the critical $E / N$ value at which one can maximize the energy transfer to $\mathrm{O}_{2}$, $(E / N)_{c}$, shifts to higher $E / N$ as the dilution rate decreases (Fig. $2 \mathrm{a} \rightarrow \mathrm{b} \rightarrow \mathrm{c}$ ); this is also true for the energy transfer to $\mathrm{H}_{2}$. Although the underlying physical and chemical effects of each balance gas are different, they all violate the chemically inert assumption. Ar* results in Penning dissociation; $\mathrm{He}^{*}$ leads to Penning ionization; $\mathrm{N}_{2} *($ both vibrational and electronic excited) is involved in dissociation reactions.

To summarize, for low temperature plasma assisted combustion, we highlighted the inevitable and significant chemical effect of a balance gas, which is generally considered as inert in combustion. First, the excited (metastable) states of a balance gas play a significant role in the chemical kinetics via collisional quenching reactions. Thus, we need to validate a reaction mechanism with various dilution rates, such that the effects of both direct electron impact reactions and indirect reactions via excited (metastable) states can be properly examined. Second, because the balance gas heavily influences the transferred electron energy to other species, the use of a practical mixture with $\mathrm{N}_{2}$ is essential. This is stressed even more by the already known significant effect of $\mathrm{N}_{2}$ excited states on the plasma chemistry [1,2,8-10]. For plasma assisted methane reforming, several kinetic studies in $\mathrm{N}_{2}$ are available [18-21]. Additionally, we showed that the dilution rate has a significant effect on the fraction of electron energy transferred to $\mathrm{H}_{2}$ and $\mathrm{O}_{2}$ as well as the critical $E / N$ where the maximum energy transfer to $\mathrm{H}_{2}$ or $\mathrm{O}_{2}$ occurs. Last but not least, when using lower dilution rates, we need to consider the temperature increase in a 0D model, because this is coupled with $E / N$. Hence, it is necessary to simultaneously adapt the temperature and $E / N$ for an accurate description of the electron energy transfer to the species in the mixture.

\section{Acknowledgements}


The research reported in this publication was funded by King Abdullah University of Science and Technology (KAUST), under award number BAS/1/1384-01-01.

\section{Supplementary materials}

File 1: Species, reduced reaction mechanism and modeling input data

\section{References}

[1] Y. Ju, W. Sun, Plasma assisted combustion: Dynamics and chemistry, Prog. Energy Combust. Sci. 48 (C) (2015) 21-83

[2] A. Starikovskiy, N. Aleksandrov, Plasma-assisted ignition and combustion, Prog. Energy Combust. Sci. 39 (1) (2013) 61-110

[3] R. Snoeckx, A. Bogaerts, Plasma technology - a novel solution for CO2 conversion?, Chem. Soc. Rev. 46 (19) (2017) 5805-63

[4] N. Tsolas, K. Togai, Z. Yin, K. Frederickson, R.A. Yetter, W.R. Lempert, et al., Plasma flow reactor studies of H2/O2/Ar kinetics, Combust. Flame 165 (2016) 144-53

[5] A. Rousso, X. Mao, Q. Chen, Y. Ju, Kinetic studies and mechanism development of plasma assisted pentane combustion, Proc. Combust. Inst. 37 (4) (2019) 5595-603

[6] H. Wang, X. You, A. V. Joshi, S.G. Davis, A. Laskin, F. Egolfopoulos, et al., USC Mech Version II. High-Temperature Combustion Reaction Model of H2/CO/C1-C4 Compounds, 2007 ,

[7] I.N. Kosarev, N.L. Aleksandrov, S.V. Kindysheva, S.M. Starikovskaia, A.Y. Starikovskii, Kinetics of ignition of saturated hydrocarbons by nonequilibrium plasma: $\mathrm{CH} 4$-containing mixtures, Combust. Flame 154 (3) (2008) 569-86

[8] N.A. Popov, Effect of a pulsed high-current discharge on hydrogen-air mixtures, Plasma Phys. Reports 34 (5) (2008) 376-91

[9] S. Kobayashi, Z. Bonaventura, F. Tholin, N.A. Popov, A. Bourdon, Study of nanosecond 
discharges in $\mathrm{H} 2$-air mixtures at atmospheric pressure for plasma assisted combustion applications, Plasma Sources Sci. Technol. 26 (7) (2017) 075004

[10] D.L. Rusterholtz, D.A. Lacoste, G.D. Stancu, D.Z. Pai, C.O. Laux, Ultrafast heating and oxygen dissociation in atmospheric pressure air by nanosecond repetitively pulsed discharges, J. Phys. D. Appl. Phys. 46 (46) (2013) 464010

[11] M. Schulze, A. Yanguas-Gil, A. von Keudell, P. Awakowicz, A robust method to measure metastable and resonant state densities from emission spectra in argon and argon-diluted low pressure plasmas, J. Phys. D. Appl. Phys. 41 (6) (2008) 065206

[12] K. Niemi, J. Waskoenig, N. Sadeghi, T. Gans, D. O’Connell, The role of helium metastable states in radio-frequency driven helium-oxygen atmospheric pressure plasma jets: measurement and numerical simulation, Plasma Sources Sci. Technol. 20 (5) (2011) 055005

[13] S. Iseni, P.J. Bruggeman, K. Weltmann, S. Reuter, Nitrogen metastable (N2(A3)) in a cold argon atmospheric pressure plasma jet : Shielding and gas composition 108 (18) (2016) 184101

[14] J.H. Kolts, D.W. Setser, Decay rates of $\operatorname{Ar}(4 s, 3 P 2), \operatorname{Ar}\left(4 s^{\prime}, 3 P 0\right), \operatorname{Kr}(5 s, 3 P 2)$, and $\operatorname{Xe}(6 s$, 3P2) atoms in argon, J. Chem. Phys. 68 (11) (1978) 4848-59

[15] J.E. Velazco, J.H. Kolts, D.W. Setser, Rate constants and quenching mechanisms for the metastable states of argon, krypton, and xenon, J. Chem. Phys. 69 (10) (1978) 4357-73

[16] G.J.M. Hagelaar, L.C. Pitchford, Solving the Boltzmann equation to obtain electron transport coefficients and rate coefficients for fluid models, Plasma Sources Sci. Technol. 14 (4) (2005) 722-33

[17] Phelps database, Available from: <www.lxcat.net/Phelps>

[18] X. Zhang, M.S. Cha, Partial oxidation of methane in a temperature-controlled dielectric barrier discharge reactor, Proc. Combust. Inst. 35 (3) (2015) 3447-54

[19] X. Zhang, M.S. Cha, Electron-induced dry reforming of methane in a temperaturecontrolled dielectric barrier discharge reactor, J. Phys. D. Appl. Phys. 46 (41) (2013) 
[20] J.-L. Liu, R. Snoeckx, M.S. Cha, Steam reforming of methane in a temperature-controlled dielectric barrier discharge reactor: the role of electron-induced chemistry versus thermochemistry, J. Phys. D. Appl. Phys. 51 (38) (2018) 385201

[21] W. Wang, R. Snoeckx, X. Zhang, M.S. Cha, A. Bogaerts, Modeling Plasma-based $\mathrm{CO}_{2}$ and $\mathrm{CH}_{4}$ Conversion in Mixtures with $\mathrm{N}_{2}, \mathrm{O}_{2}$, and $\mathrm{H}_{2} \mathrm{O}$ : The Bigger Plasma Chemistry Picture, J. Phys. Chem. C (2018) acs.jpcc.7b10619 